\begin{tabular}{ll} 
Abstract 0017 Table 1 & Chemsex-related admissions \\
\hline Diagnosis & N (\%) \\
\hline Overdose & $9(47.4 \%), 4$ ITU admissions \\
Psychosis & $3(15.8 \%)$ \\
Abscess & $2(10.5 \%)$ \\
Arrhythmias & $2(10.5 \%)$ \\
DVTs & $1(5.3 \%)$ \\
Withdrawal & $1(5.3 \%)$ \\
Rhabdomyalysis & $1(5.3 \%)$ \\
\hline
\end{tabular}

GHB, 5 (26.3\%) mephedrone and 4 (21.1\%) crystal meth. Cause of admission can be seen in Table 1 . There were 3 deaths due to drug overdoses during the study period.

Discussion/conclusion RDU was responsible for 9.8\% of admissions, with GHB, mephedrone and crystal meth responsible for 21-26\%. This may underestimate the true effect of drug admissions as it only involves HIV positive MSM. We've developed a chemsex clinic and city-wide task and finish group, in liaison with Public Health to address the growing effect of chemsex. Clinicians need to ensure RDU is regularly reviewed and timely interventions are offered to limit harms.

\section{COMMUNITY VIRAL LOAD: A NEW POPULATION-BASED BIOMARKER OF HIV DISEASE BURDEN IN SCOTLAND}

${ }^{1}$ Muhammad Ismail, ${ }^{2}$ Daniela Brawley*, ${ }^{2}$ Steve Baguley. 'University of Aberdeen School of Medicine and Dentistry, Aberdeen, UK; ${ }^{2}$ NHS Grampian Sexual Health Services, Grampian, UK

\subsection{6/sextrans-2016-052718.17}

Background/introduction "Community viral load" (CVL) refers to an aggregate biological measure of viral load (VL) for a particular geographic location. Studies have suggested that CVL may be used as a population-based biomarker for HIV transmission, and that its reduction is associated with a decrease in HIV incidence. Currently, there is no published data on CVL in Scotland. Aim(s)/objectives This study aims to measure CVL and to estimate the HIV transmission potential of communities in Scotland. Methods HIV/AIDS surveillance data on patient demographics, first VL in 2014, and region of residence were analysed. Mean CVL was measured as the arithmetic average and total CVL the arithmetic sum of all VL in our data set respectively. Statistical analyses were performed using SPSS 23 at 95\% significance level. Shapiro-Wilk test was performed for normality. Chi-square analysis and Kruskal-Wallis test were performed for differences in variables. Spearman's correlation was performed for correlations between CVL and HIV incidence.

Results 4126 non-duplicate cases were analysed. Mean CVL was highest in Central South-West (CSW) $(\mu=20,469,95 \%$ CI $=$ 8146-32,933), followed by Central South-East (CSE) and North respectively. There was a significant difference in mean rank CVL between North-CSW and North-CSE. There was a positive correlation between mean CVL and HIV quarterly incidence for CSW (Spearman's rho $=0.062, \mathrm{p}=0.01$ ) and CSE (Spearman's rho $=0.032, \mathrm{p}=0.196)$, whereas a negative correlation was seen in North (Spearman's rho $=-0.047, \mathrm{p}=0.202$ ).

Discussion/conclusion This study highlights the relationship between CVL and HIV quarterly incidence in Scotland in 2014.
Further work using annual incidence data is needed to verify these conclusions and to determine factors influencing these results.

\section{EXTRA-GENITAL SAMPLES FOR GONORRHOEA AND CHLAMYDIA IN WOMEN AND MSM: SELF-TAKEN SAMPLES ANALYSED SEPARATELY COMPARED WITH SELF-TAKEN POOLED SAMPLES}

${ }^{1}$ Janet Wilson* ${ }^{*}{ }^{1}$ Harriet Wallace, ${ }^{1}$ Michelle Loftus-Keeling, ${ }^{2}$ Helen Ward, ${ }^{3}$ Claire Hulme ${ }^{4}$ Mark Wilcox. 'Leeds Sexual Health, Leeds Teaching Hospitals NHS Trust, Leeds, UK; ${ }^{2}$ Department of Infectious Disease Epidemiology, Imperial College, London, UK; ${ }^{3}$ Academic Unit of Health Economics, University of Leeds, Leeds, UK; ${ }^{4}$ Department of Clinical Microbiology, Leeds Teaching Hospitals NHS Trust, Leeds, UK

\subsection{6/sextrans-2016-052718.18}

Background Extra-genital infections are common in MSM and women and are frequently the sole sites of infection. However, analysing samples from the rectum and pharynx, in addition to the urogenital tract, trebles the diagnostic cost.

Aim Can samples from three sites be pooled into one NAAT container and still achieve the same sensitivity and specificity as the samples analysed separately?

Methods Women and MSM attending a sexual health clinic were invited into a 'swab yourself' trial. Two self-taken samples (one for separate analysis and one for pooling) were taken from the pharynx and rectum with VVS in women and FCU in MSM. The sampling order of the pooled or analysed separately swabs was randomised. Gonorrhoea (NG) and chlamydia (CT) were diagnosed using NAATs. Patient infected status was defined as at least two positive confirmed samples.

Results 1251 women and MSM were recruited to January 2016. Overall prevalence of infections was NG 5.7\% and CT $17.8 \%$. Sensitivity, specificity, PPV and NPV are shown in the table:

Conclusion This on-going study demonstrates that self-taken samples from the rectum, pharynx and urogenital tract are comparable in sensitivity and specificity if analysed separately or as a pooled sample. In MSM the diagnostic costs of three separate analyses are unaffordable for many health systems but a pooled sample has the same laboratory cost as a urogenital sample. These findings mean triple site testing could be expanded into women at no additional health service cost.

Abstract 0019 Table 1 Sensitivity \& specificity of separate and pooled samples

\begin{tabular}{lllll}
\hline & $\begin{array}{l}\text { Sensitivity } \\
(95 \% \mathrm{Cl})\end{array}$ & $\begin{array}{l}\text { Specificity } \\
(95 \% \mathrm{Cl})\end{array}$ & $\begin{array}{l}\text { PPV } \\
(95 \% \mathrm{Cl})\end{array}$ & $\begin{array}{l}\text { NPV } \\
(95 \% \mathrm{Cl})\end{array}$ \\
\hline NG separate samples & 98.6 & 99.9 & 98.6 & 99.9 \\
& $(90.2-99.7)$ & $(99.5-100.0)$ & $(92.6-100.0)$ & $(99.5-100.0)$ \\
NG pooled & 97.2 & 99.9 & 98.6 & 99.8 \\
& $(90.2-99.7)$ & $(99.5-100.0)$ & $(92.3-100.0)$ & $(99.4-100.0)$ \\
CT separate samples & 99.1 & 99.7 & 98.7 & 99.8 \\
& $(96.8-99.4)$ & $(99.2-99.9)$ & $(96.1-99.7)$ & $(99.3-100.0)$ \\
CT pooled & 95.5 & 99.5 & 97.7 & 99.0 \\
& $(91.9-97.8)$ & $(98.9-99.8)$ & $(94.7-99.3)$ & $(98.2-99.5)$
\end{tabular}

There was no difference between self-taken samples analysed separately or pooled by McNemar test. 